\title{
Microdiscectomy for the Treatment of Lumbar Disc Herniation: An Evaluation of Reoperations and Long-Term Outcomes
}

\author{
Alexander Aichmair ${ }^{1}$ Jerry Y. Du ${ }^{1} \quad$ Jennifer Shue ${ }^{1} \quad$ Gisberto Evangelisti $^{2}$ Andrew A. Sama ${ }^{1}$ \\ Alexander P. Hughes ${ }^{1}$ Darren R. Lebl ${ }^{1}$ Jayme C. Burket ${ }^{3} \quad$ Frank P. Cammisa $^{1}$ Federico P. Girardi ${ }^{1}$ \\ ${ }^{1}$ Department of Orthopaedic Surgery, Spine and Scoliosis Service, \\ Hospital for Special Surgery, Weill Cornell Medical College, New York, \\ New York, United States \\ 2 st Orthopaedic Clinic, Cisanello Hospital, University of Pisa, Pisa, Italy

\begin{abstract}
Address for correspondence Alexander Aichmair, MD, Department of Orthopaedic Surgery, Spine and Scoliosis Service, Hospital for Special Surgery, Weill Cornell Medical College, 535 East 70th Street, New York, NY 10021, United States (e-mail: AichmairA@gmail.com).
\end{abstract}

${ }^{3}$ Department of Epidemiology and Biostatistics, Hospital for Special Surgery, Weill Cornell Medical College, New York, New York, United States

Evid Based Spine Care J 2014;5:77-86.

\begin{abstract}
Keywords

- microdiscectomy

- limited discectomy

- long-term outcome

- reoperation

- Oswestry disability index

- ODI

- lumbar disc herniation

Design Retrospective case series.

Objective The objective of this study was to assess the reoperation rate after microdiscectomy for the treatment of lumbar disc herniation (LDH) in patients with $\geq 5$-year follow-up and identify demographic, perioperative, and outcome-related differences between patients with and without a reoperation.

Methods The medical records, operative reports, and office notes of patients who had undergone microdiscectomy at a single institution between March 1994 and December 2007 were reviewed and long-term follow-up was assessed via a telephone questionnaire.

Results Forty patients (M:24, F:16) with an average age at surgery of $39.9 \pm 12.5$ years (range: 18-80) underwent microdiscectomy at the levels L5-S1 ( $n=28,70 \%)$, L4-L5 ( $n=9,22.5 \%), \mathrm{L} 3-\mathrm{L} 4$ ( $n=2,5.0 \%)$, and L1-L2 $(n=1,2.5 \%)$. After an average of $40.4 \pm 40.1$ months (range: $1-128$ ), $25 \%$ of patients (10/40) required further spine surgery related to the initial microdiscectomy. At an average postoperative follow-up of $11.1 \pm 4.0$ years (range: $5-19$ ), additional symptoms apart from back and leg pain were reported more frequently by patients who underwent a reoperation $(p=0.005)$. Patient satisfaction was significantly higher in patients who did not undergo a reoperation $(p=0.041)$. For the Oswestry disability index, pain intensity $(p=0.036)$, and pain-related sleep disturbances $(p=0.006)$ were reported to be more severe in the reoperation group.

Conclusions Microdiscectomy for the treatment of LDH results in a favorable longterm outcome in the majority of cases. The reoperation rate was higher in our series than reported in previous investigations with shorter follow-up. Although there were no statistically significant pre-/perioperative differences between patients with and without reoperation, our findings suggest a difference in self-reported long-term outcome measures.
\end{abstract}

received

November 13, 2013 accepted after revision June 23,2014 (c) 2014 Georg Thieme Verlag KG Stuttgart · New York
DOI http://dx.doi.org/ 10.1055/s-0034-1386750. ISSN 1663-7976. 


\begin{tabular}{|l|l|}
\hline Final level of evidence (LoE)-prognostic & \\
\hline Study design & \\
\hline Prospective cohort & \\
\hline Retrospective cohort & \\
\hline Case-control & \\
\hline Case series & $\mathrm{x}$ \\
\hline Methods & \\
\hline Patients at similar point in course of treatment & $\mathrm{x}$ \\
\hline F/U $\geq 85 \%$ & \\
\hline Similarity of treatment protocols for patient groups & $\mathrm{x}$ \\
\hline $\begin{array}{l}\text { Patients followed for long enough for } \\
\text { outcomes to occur }\end{array}$ & $\mathrm{x}$ \\
\hline Control for extraneous risk factors & \\
\hline Overall level of evidence & $\mathrm{IV}$ \\
\hline
\end{tabular}

The definitions of the different levels of evidence are available on page 166 .

\section{Study Rationale}

Lower back pain (LBP) is the most frequently reported type of pain, affecting $29 \%$ of the adult population in the United States, ${ }^{1}$ resulting in an estimated $\$ 84.1$ to $\$ 624.8$ billion total costs for the U.S. health care system. ${ }^{2}$ Apart from nonspecific back pain, degenerative disease and disc herniation represent the most common causes for LBP-related physician office consultations. ${ }^{3}$ In fact, the majority of lumbar radicular pain symptoms is the result of a disc herniation, defined as bulging of the nucleus pulposus through a fissure or tear within the annulus fibrosus. ${ }^{4}$ Although favorable outcomes have been demonstrated for both surgical and nonoperative treatment options, patients who underwent open discectomy for lumbar disc herniation (LDH) were shown to have better selfreported outcomes than conservatively treated individuals. ${ }^{5}$

Due to the postulated advantages of reduced tissue invasiveness, limited blood loss, a shorter duration of surgery, and a faster postoperative recovery, minimally invasive microdiscectomy, often also referred to as limited discectomy, has been established as an alternative to traditional, more aggressive open approaches in the treatment of LDHs. ${ }^{6}$ Nevertheless, based on previous study findings, controversies regarding the benefits of a minimally invasive over an open surgical approach remain. ${ }^{7-10}$ In a recently published long-term outcome analysis, limited microdiscectomy was reported to be an effective surgical approach for the treatment of LDH. However, the reported disc herniation recurrence rate was higher compared with previous studies with a shorter follow-up period, ${ }^{8}$ which warrants further follow-up investigations to assess the long-term efficacy of this minimally invasive surgical approach.

\section{Objective}

The objective of this study was to assess the reoperation rate after microdiscectomy for the treatment of LDH in patients with $\geq 5$-year follow-up and identify demographic, periop- erative, and outcome-related differences between patients with and without a reoperation.

\section{Methods}

\section{Study Population}

After obtaining the approval of the Institutional Review Board, the medical records, physician office charts, operative reports, and radiographic imaging studies of patients who had undergone minimally invasive microdiscectomy at a single institution between March 1994 and December 2007 were retrospectively reviewed. All patients underwent surgery for the treatment of single-level LDH in the setting of degenerative disc disease with concomitant nerve root compression and radicular symptoms. The exclusion criteria of the present study were defined as (1) a follow-up period of less than 5 years and (2) microdiscectomy as a revision surgery (see PPO table in online supplementary material).

\section{Baseline/Perioperative Characteristics}

Data collection included gender, age at index surgery, body mass index (BMI), preoperative neurological examination, and length of stay (-Table $\mathbf{1}$ ). The sensory and motor neurological function was assessed both in the pre- and the postoperative setting by a fellowship-trained spine surgeon. A sensory deficit was defined as any change in the individual's sensory neurological function, including numbness, para-/dysesthesias, and sensation of pins and needles, and a motor deficit was defined as any change in the individual's muscle strength during the neurological examination.

Furthermore, information about the preoperative visual analog scale (VAS) scores of back and lower extremity pain were retrieved from the medical records. If the appropriate score was not specified by the patient, the documented characterization of pain severity was used to estimate the VAS score: "minimal," "mild," "moderate," "quite significant," "quite strong," and "severe" pain was defined as a VAS score of $1.5,3,6,7,8$, and 9 , respectively (preoperative back and leg pain estimation in $12.5 \%$ [ $n=5]$ and $20.0 \%$ [ $n=8]$ of patients, respectively).

Surgery-related data were collected on previous surgeries of the lumbar spine, surgically addressed levels, intraoperative complications, postoperative clinical outcome, and any requirements for add-on lumbar spine surgeries.

\section{Surgical Technique}

All included patients underwent surgery for single-level LDH with radicular lower extremity symptoms. Minimally invasive microdiscectomy at the appropriate level was performed according to a standardized institutional surgical protocol. In brief summary, general anesthesia was induced, the patient secured in prone position to the surgical table, and the spine approached via a posterior midline incision. After the removal of the ligamentum flavum at the appropriate motion segment level, the compressed nerve root was retracted and protected to safely remove all extruded disc segments. When indicated, the annular lesion was further inspected and 
Table 1 Descriptive patient characteristics $(n=40)$

\begin{tabular}{|c|c|c|c|c|}
\hline Variable & Mean or $N$ & SD or $\%$ & Min & $\operatorname{Max}$ \\
\hline Age at surgery $(\mathrm{y})$ & 39.9 & 12.5 & 18 & 80 \\
\hline \multicolumn{5}{|l|}{ Gender } \\
\hline Female & 16 & $40.0 \%$ & & \\
\hline Male & 24 & $60.0 \%$ & & \\
\hline BMI $\left(\mathrm{kg} / \mathrm{m}^{2}\right)$ & 26.8 & 4.1 & 19.0 & 35.9 \\
\hline \multicolumn{5}{|l|}{ Neurological exam } \\
\hline Preoperative VAS (back) & 4.8 & 3.2 & 0 & 10 \\
\hline Preoperative VAS (leg) & 6.5 & 2.1 & 2 & 10 \\
\hline \multicolumn{5}{|l|}{ Preoperative sensory deficit } \\
\hline No & 9 & $22.5 \%$ & & \\
\hline Yes & 31 & $77.5 \%$ & & \\
\hline \multicolumn{5}{|l|}{ Preoperative motor deficit } \\
\hline No & 12 & $30.0 \%$ & & \\
\hline Yes & 28 & $70.0 \%$ & & \\
\hline \multicolumn{5}{|l|}{ Microdiscectomy level } \\
\hline $\mathrm{L} 1-2$ & 1 & $2.5 \%$ & & \\
\hline L2-3 & 0 & $0.0 \%$ & & \\
\hline L3-4 & 2 & $5.0 \%$ & & \\
\hline L4-5 & 9 & $22.5 \%$ & & \\
\hline L5-S1 & 28 & $70.0 \%$ & & \\
\hline Length of surgery (min) & 77.9 & 36.8 & 43 & 256 \\
\hline Days of hospitalization & 1.7 & 1.2 & 1 & 7 \\
\hline
\end{tabular}

Abbreviations: SD, standard deviation; VAS, visual analog scale.

additional disc fragments removed. Once the nerve root was found to be free of compression, the surgical field was copiously irrigated and the wound closed. Finally, the patient was awakened from anesthesia and transferred to the recovery room, after the motor strength of the lower extremities had been evaluated.

\section{Postoperative Clinical Outcome}

Data on the short-term postoperative clinical outcome were retrieved from the patients' medical records, and office notes, including the presence of residual radicular pain, sensory, and motor deficits, as well as the need for additional lumbar spinal surgery involving the level of the initial microdiscectomy.

\section{Long-Term Clinical Outcome}

Patients were called to assess their postoperative long-term clinical outcome after a follow-up of at least 5 years after the index microdiscectomy. In addition to the utilized telephone questionnaire, the Oswestry disability index (ODI) score ${ }^{11}$ was obtained for each patient. Patients who were not available for the telephone follow-up interview were considered as "lost to follow-up."
Postoperative long-term outcome parameters that were assessed via the telephone questionnaire included (1) the need for another back surgery after the microdiscectomy and (2) whether the additional surgery was related to the index surgery, (3) the need for pain management measures after the index surgery, (4) the back and leg pain severity at the time of the telephone follow-up graded according to the VAS scoring system, (5) the presence of additional symptoms apart from pain such as sensory and motor deficits reported on a scale from 0 to 10 with 0 being no additional symptoms and 10 being the highest severity of additional symptoms, (6) the degree of improvement as a result of the index microdiscectomy, (7) the degree of satisfaction regarding the outcome of the surgery reported on a scale from 0 to 10 with 0 being complete dissatisfaction and 10 being complete satisfaction, (8) the patient's opinion regarding undergoing the same surgery again, (9) the ability to work after surgery, (10) the present working status, and (11) the Oswestry low back pain disability index (ODI)-Version 1.0.

The used ODI questionnaire consisted of 10 questions regarding pain intensity and the degree of pain over time, as well as the ability to perform personal care tasks, lift weights, walk, sit, stand, sleep, travel, and participate in social 
life. The percentage of disability was then calculated based on the patient's total ODI score divided by the maximally achievable score (i.e., 50) for each patient.

\section{Statistical Analysis}

The results of descriptive data analysis are shown as means \pm standard deviations for continuous variables, and as frequencies and percentages for categorical variables. Differences in pre- and postoperative VAS scores were assessed with paired $t$-tests. The association between each independent risk factor and the requirement for additional lumbar spine surgery related to the index microdiscectomy was then assessed using a bivariate analysis. Due to the small sample size, we were unable to perform a multivariate analysis to control for potential confounding when comparing outcomes between those with and without an additional lumbar surgery. Independent sample $t$-tests or nonparametric Mann-Whitney $U$ tests were used for continuous risk factors, while chi-squared or Fisher exact tests were used for categorical risk factors. Statistical significance was defined at the level of $\alpha=0.05$. Data analysis was performed using SAS software version 9.3 (SAS Institute Inc., Cary, North Carolina, United States).

\section{Results}

\section{Study Population}

Out of 203 microdiscectomy patients with a postoperative follow-up of at least 5 years, 8 patients did not meet the inclusion criteria of the present study, due to the index microdiscectomy as a revision surgery (-Table $\mathbf{1}$ ). Out of the remaining 195 eligible patients, 1 patient refused to participate in the present study, resulting in a total of 194 potential subjects for follow-up. Forty-nine patients had incomplete data, and another 105 patients were not available for the telephone follow-up interview, resulting in a total of 40 patients (16 females, 24 males) available for analysis. The average patient age at surgery was $39.9 \pm 12.5$ years (range: $18-80$ ), and the average BMI was $26.8 \pm 4.1$ $\mathrm{kg} / \mathrm{m}^{2}$ (range: $19.0-35.9$ ) (-Fig. 1). During the preoperative neurological examination, all patients complained of lower back and/or lower extremity pain. Preoperative VAS scores for lower back and lower extremity pain were reported to be $4.8 \pm 3.2$ out of 10 (range: $0-10$ ), and $6.5 \pm 2.1$ out of 10 (range: 2-10), respectively. Preoperative neurological examination of the study population revealed a sensory deficit in $77.5 \%(n=31)$ and a motor deficit in $70 \%(n=28)$ of cases.

\section{Surgical Details}

Single-level lumbar microdiscectomy was performed by one of three fellowship-trained spine surgeons at the levels L5-S1 ( $n=28,70 \%), \mathrm{L} 4-\mathrm{L} 5$ ( $n=9,22.5 \%), \mathrm{L} 3-\mathrm{L} 4(n=2,5.0 \%)$, and L1-L2 ( $n=1,2.5 \%)$. There were no patients with a surgery performed at the L2-L3 level. There were no cases of a surgical procedure previously performed at the index microdiscectomy level. The average length of surgery was $77.9 \pm 36.8$ minutes (range: 43.0-256.0) with no reports of intra- operative complications, and the patients were discharged after an average of $1.7 \pm 1.2$ days (range: $1-7$ ).

\section{Postoperative Clinical Outcome}

In the early postoperative period $(1.5 \pm 1.0$ months postoperatively on average, range: $0.2-6.3), 62.2 \%$ (23/37) of patients presented with residual radicular pain, 25.7\% (9/35) with a sensory deficit, and $8.1 \%$ (3/37) with a residual motor deficit, respectively. After an average of $40.4 \pm 40.1$ months (range: $1-128), 25 \%$ of patients (10/40) required further surgery at the lumbar spine, which involved the level of the initial microdiscectomy. Out of 30 patients who did not undergo additional spine surgery, 10 patients (33.3\%) needed further pain management measures.

\section{Long-Term Clinical Outcome}

At the time of the long-term telephone follow-up, which was conducted after an average of $11.1 \pm 4.0$ years (range: $5-19$ ) after the microdiscectomy, the VAS scores for back and leg pain were reported to be $2.1 \pm 1.9$ out of 10 (range: $0-9$ ), and $1.6 \pm 1.6$ out of 10 (range: $0-8$ ), respectively, which was significantly lower compared with the preoperative VAS scores $(p<0.001$ and $p<0.001$ ) (-Fig. 2). The pre- to postoperative changes in the back and leg pain VAS scores were $-2.7 \pm 3.7$ (range: $-9-5$ ) and $-4.9 \pm 2.6$ (range: -9-1), respectively. The severity of other reported symptoms in the back and lower extremities apart from pain were described as $1.7 \pm 1.7$ (range: $0-7$ ) on average.

Improvement of symptoms in the postoperative setting compared with the preoperative condition was stated by 37 out of 40 patients (92.5\%). The majority of patients $(31 / 40$, $77.5 \%$ ) reported "a lot of" improvement after surgery, whereas "moderate" or "little" improvement was reported in 5\% (2/ $40)$ and $10 \%$ (4/40) of patients, respectively. No improvement

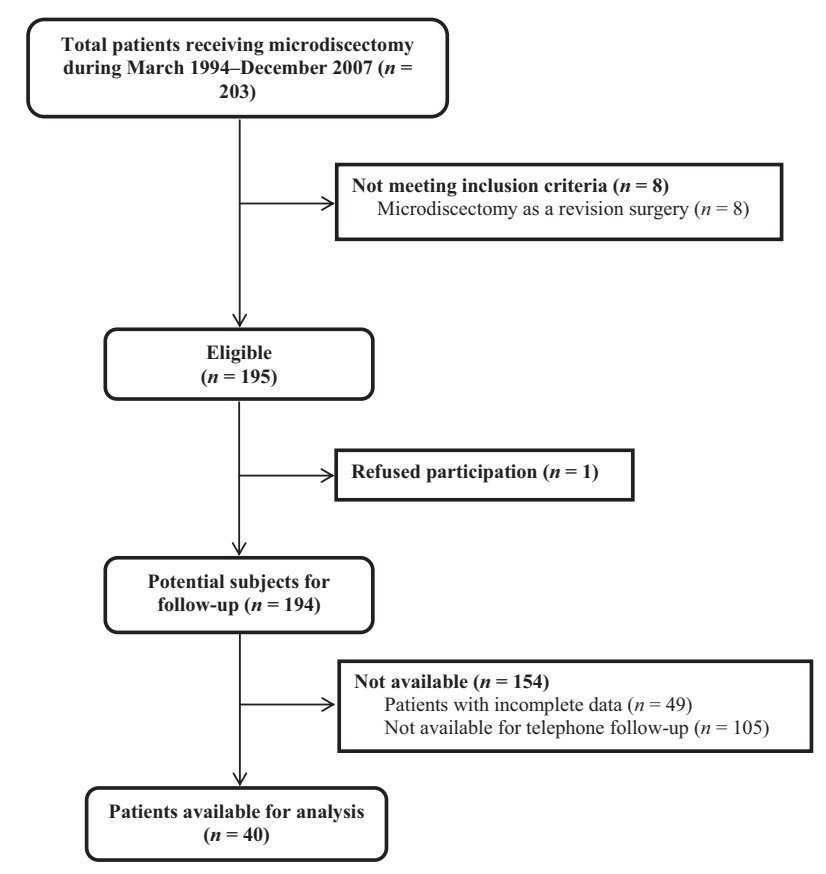

Fig. 1 Patient sampling and selection. 


\section{Visual Analogue Scale (VAS)}

Back Pain

Leg Pain

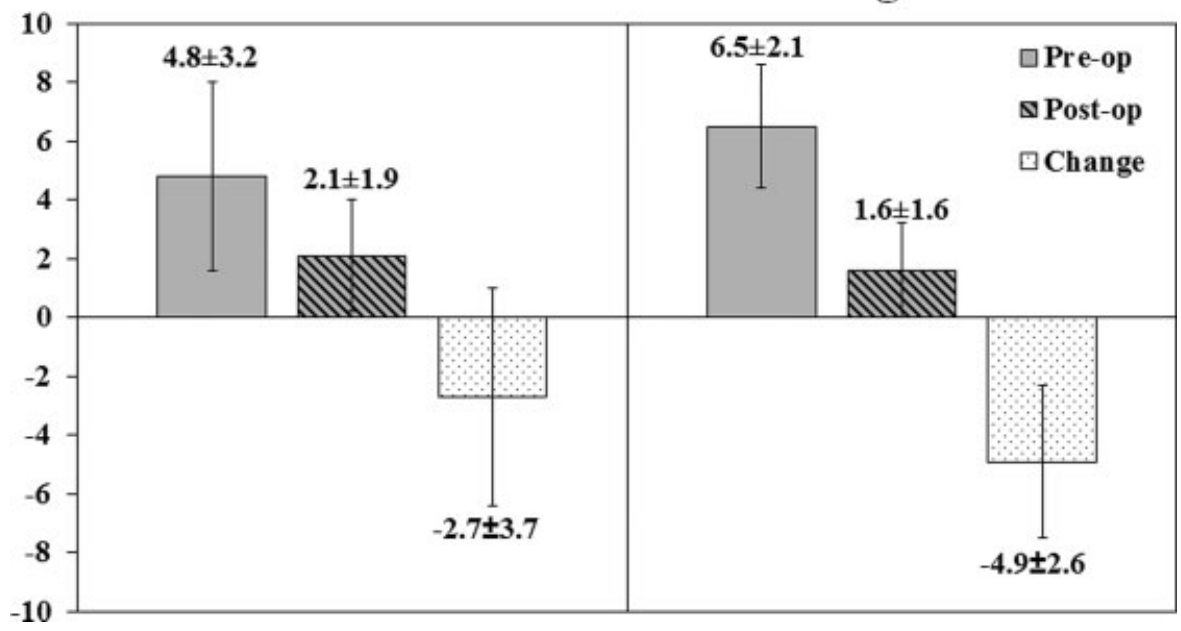

Fig. 2 At postoperative follow-up of $11.1 \pm 4.0$ years (range: $5-19$ ), the VAS scores for both back and leg pain were significantly lower, compared with the preoperative setting ( $p<0.001$ and $p<0.001$, respectively). The pre- to postoperative changes in the back and leg pain VAS scores were $-2.7 \pm 3.7$ (range: -9 to 5 ) and $-4.9 \pm 2.6$ (range: -9 to 1 ), respectively.

or worsening of radiculopathy was reported in $2.5 \%(1 / 40)$ and $5 \%(2 / 40)$ of patients, respectively. In terms of long-term overall patient satisfaction, the average value was $9.1 \pm 2.1$ (range: $0-10$ ), and $95.0 \%$ of patients (38/40) stated they would undergo the same surgery again. Furthermore, 9 patients $(22.5 \%)$ were unable to work with respect to their spine condition, with 27 patients (67.5\%) being employed at the time of responding to the questionnaire. In only 1 of the 13 unemployment cases, the reason for unemployment was related to the individual's spine condition. The results of the ODI questionnaire indicated an average of $15.4 \pm 20.7 \%$ (range: $0-80$ ) at the time of the telephone follow-up, which can be considered as "minimal disability."11

\section{Comparison of Patients with and without Additional Spine Surgery}

The results of the detailed subanalysis comparing patients with and without additional spine surgery are shown in - Table 2. In summary, there were no statistically significant differences between both study subpopulations, with regard to pre- and perioperative clinical data, only with regard to the postoperative long-term clinical outcome. In the reoperation subcohort, the reported outcome data were assessed at an average of $74.7 \pm 52.5$ months (range: $14-201$ ) after the revision surgery.

Additional symptoms in the back and legs apart from pain were reported more frequently by patients who had undergone additional spine surgery after the initial microdiscectomy, which was statistically significant $(p=0.005)$. Furthermore, patient satisfaction was reported to be significantly higher in patients who had not undergone a reoperation $(p=0.041)$. Although not statistically significant, there was a trend of both higher pre- and postoperative VAS back pain scores in the reoperation study subpopulation
( $p=0.082, p=0.117$ ). The pre-to-postoperative change in the average VAS back pain scores was comparable between patients with and without a reoperation $(-3.4 \pm 4.8$ and $-2.5 \pm 3.2$ points difference, respectively; $p=0.495$ ), which was also the case for the pre-to-postoperative change in the average VAS leg pain scores $(-4.7 \pm 3.4$ and $-5.0 \pm 2.4$ points difference, respectively; $p=0.950$ ). Furthermore, the L4-L5 level was more frequently addressed in the reoperation group (40 vs. 17\%); however, this observed difference was not statistically significant $(p=0.100)$.

With regard to the ODI questionnaire, the pain intensity was more severe in the reoperation group $(p=0.036)$. Furthermore, the severity of pain-related sleep disturbances was significantly higher in patients who had undergone add-on surgery $(p=0.006)$. Although not statistically significant, there was a trend of a more severe pain-related inability to travel ( $p=0.092$ ) in patients who had undergone a reoperation, in addition to a trend toward a higher total ODI score $(24.6 \pm 25.7$ vs. $12.3 \pm 18.2 \%, p=0.065)$.

\section{Discussion}

Minimally invasive microdiscectomy has been established as an alternative to traditional, more aggressive open approaches for the treatment of $\mathrm{LDH}$, which is based on proposed advantages including reduced tissue invasiveness, limited blood loss, a shorter duration of surgery, and a faster postoperative recovery. ${ }^{6-10}$ Although microdiscectomy was previously suggested to result in a favorable postoperative outcome, the reported rate of recurrent disc herniations was higher in some long-term follow-up studies compared with investigations with a shorter follow-up period..$^{8,12}$ Therefore, the aim of the present study was to evaluate the rate of revision surgery, as well as the long-term outcome after 
82 Microdiscectomy for the Treatment of Lumbar Disc Herniation Aichmair et al.

Table 2 Comparison of patients with or without reoperation after microdiscectomy

\begin{tabular}{|c|c|c|c|c|c|c|c|}
\hline \multirow[b]{2}{*}{ Variable } & \multicolumn{3}{|c|}{ No add-on surgery $(n=30)$} & \multicolumn{3}{|c|}{ Add-on surgery $(n=10)$} & \multirow[b]{2}{*}{$p$ value } \\
\hline & $N$ & Mean or \% & SD & $N$ & Mean or \% & SD & \\
\hline Age at surgery (y) & & 40.3 & 12.9 & & 38.8 & 11.7 & 0.595 \\
\hline Male gender & 19 & $63 \%$ & & 5 & $50 \%$ & & 0.482 \\
\hline $\mathrm{BMI}\left(\mathrm{kg} / \mathrm{m}^{2}\right)$ & & 26.7 & 3.8 & & 27.1 & 4.9 & 0.820 \\
\hline \multicolumn{8}{|l|}{ Neurological exam } \\
\hline Preoperative VAS (back) & & 4.3 & 2.9 & & 6.3 & 3.7 & 0.082 \\
\hline Preoperative VAS (leg) & & 6.4 & 2.0 & & 6.6 & 2.6 & 0.883 \\
\hline Preoperative sensory deficit & & & & & & & 0.999 \\
\hline No & 7 & $23 \%$ & & 2 & $20 \%$ & & \\
\hline Yes & 23 & $77 \%$ & & 8 & $80 \%$ & & \\
\hline Preoperative motor deficit & & & & & & & 0.693 \\
\hline No & 10 & $33 \%$ & & 2 & $20 \%$ & & \\
\hline Yes & 20 & $67 \%$ & & 8 & $80 \%$ & & \\
\hline Microdiscectomy level & & & & & & & 0.100 \\
\hline $\mathrm{L} 1-2$ & 0 & $0 \%$ & & 1 & $10 \%$ & & \\
\hline $\mathrm{L} 2-3$ & 0 & $0 \%$ & & 0 & $0 \%$ & & \\
\hline L3-4 & 2 & $6.7 \%$ & & 0 & $0 \%$ & & \\
\hline L4-5 & 5 & $17 \%$ & & 4 & $40 \%$ & & \\
\hline L5-S1 & 23 & $77 \%$ & & 5 & $50 \%$ & & \\
\hline Length of surgery (min) & & 77.4 & 40 & & 79.9 & 23.2 & 0.300 \\
\hline Days of hospitalization & & 1.5 & 1.0 & & 2.1 & 1.8 & 0.184 \\
\hline \multicolumn{8}{|l|}{ Telephone follow-up } \\
\hline Postoperative VAS (back) & & 1.8 & 1.7 & & 2.9 & 2.1 & 0.117 \\
\hline Change from baseline VAS back & & -2.5 & 3.2 & & -3.4 & 4.8 & 0.495 \\
\hline Postoperative VAS (leg) & & 1.5 & 1.5 & & 1.9 & 1.6 & 0.295 \\
\hline Change from baseline VAS leg & & -5.0 & 2.4 & & -4.7 & 3.4 & 0.950 \\
\hline Additional symptoms & & 1.2 & 1.0 & & 3.3 & 2.3 & 0.005 \\
\hline Patient satisfaction & & 9.6 & 1.1 & & 8.0 & 3.5 & 0.041 \\
\hline Ability to work & & & & & & & 0.655 \\
\hline No & 6 & $20 \%$ & & 3 & $30 \%$ & & \\
\hline Yes & 24 & $80 \%$ & & 7 & $70 \%$ & & \\
\hline Oswestry disability index (\%) & & $12.3 \%$ & $18.2 \%$ & & $24.6 \%$ & $25.7 \%$ & 0.065 \\
\hline 1. Pain intensity $(0-5)$ & & 0.5 & 1.2 & & 1.5 & 1.8 & 0.036 \\
\hline 2. Personal care $(0-5)$ & & 0.3 & 0.9 & & 0.4 & 1.0 & 0.625 \\
\hline 3. Lifting $(0-5)$ & & 0.8 & 1.5 & & 2.1 & 2.2 & 0.302 \\
\hline 4. Walking $(0-5)$ & & 0.4 & 1.0 & & 0.6 & 1.3 & 0.282 \\
\hline 5. Sitting $(0-5)$ & & 0.8 & 1.2 & & 0.9 & 1.3 & 0.959 \\
\hline 6. Standing $(0-5)$ & & 0.7 & 1.3 & & 1.4 & 1.8 & 0.252 \\
\hline 7. Sleeping (0-5) & & 0.3 & 0.9 & & 1.5 & 1.8 & 0.006 \\
\hline 8. Social life $(0-5)$ & & 0.5 & 1.2 & & 1.0 & 1.4 & 0.377 \\
\hline 9. Traveling (0-5) & & 0.6 & 1.0 & & 1.0 & 1.7 & 0.092 \\
\hline 10. Changing pain $(0-5)$ & & 1.2 & 1.7 & & 1.9 & 1.9 & 0.261 \\
\hline
\end{tabular}


microdiscectomy for the treatment of LDH in patients with a minimum postoperative follow-up of 5 years. An illustrative case presentation of a reoperation is shown in - Fig. 3A-D.

In their previously published meta-analysis on recurrent disc herniations after disc removal, McGirt et al reviewed 54 studies including a total of 13,359 patients and compared the postoperative outcomes after a limited versus an aggressive approach to lumbar discectomy. In their subanalysis assessing persistent or recurrent back/leg pain after a minimum of 2 years postoperatively, the authors reported a cumulative incidence of $11.6 \%$ (range: $7-16 \%$ ) for limited versus $27.9 \%$ (range: 19-36\%) for aggressive discectomy. ${ }^{12}$ According to the results of the present study, the rate of reoperation related to the index microdiscectomy was $25 \%$, which is higher than the recently reported rate of $18.5 \%$ by Soliman et al. However, the average postoperative follow-up period of the quoted study was 7.2 years, which is approximately 3.5 years shorter than in the present study. ${ }^{8}$ According to the findings of a recent prospective cohort study on 108 patients, a large size of the annular defect and little removal of disc material were identified as risk factors for a same-level recurrent disc herniation after lumbar discectomy. However, discectomy was performed by five surgeons, each from a distinct institution, according to a surgeon-preference based, nonstandardized protocol, ranging from removal of the herniated fragment only to intradiscal curettage with subtotal discectomy. ${ }^{13}$ Their results are similar to the findings of Carragee et al, who identified the lowest reherniation and reoperation rates for patients with a disc fragment and a small annular fissure, both reported to be $1 \%{ }^{14}$ In their retrospective analysis, Yorimitsu et al highlighted the favorable long-term outcome after standard discectomy for the treatment of $\mathrm{LDH}$. According to their findings regarding the activities of daily living, assessed utilizing the Japanese Orthopedic Association scoring system, the degree of disability was higher for activities such as leaning forward and lifting heavy objects compared with others. ${ }^{15}$

In the present study population, a reoperation was performed after an average interval of $40.4 \pm 40.1$ months (range: 1-128). In only 2 out of 10 cases, the reoperation was performed within the first 12 months and in only 3 out of 10 cases within the first 24 months after surgery. As already suggested in a previous study, ${ }^{8}$ it is hard to define whether a reoperation was necessary due to inefficacy of the initial surgical attempt, or due to the progression of the degenerative spine disease itself. We believe that in the study subjects with a reoperation after an interval of more than 24 months, degenerative disease progression was the predominant cause for symptom recurrence as opposed to surgical failure. By comparing patients with and without a reoperation after the index microdiscectomy, we were able to identify a difference in the postoperative long-term outcome. According to our data, statistically significant differences between both study subgroups were observed regarding the presence of additional symptoms apart from back or leg pain, the degree of patient satisfaction, the severity of pain-related sleep disturbances, and the patient-reported pain intensity as scored according to the
ODI in the postoperative setting. Nevertheless, it needs to be highlighted that it remains unclear whether these differences were observed because of the performance of an additional lumbar spine surgery or a more advanced disease at baseline in the reoperation subcohort, as reflected by a trend toward a higher preoperative back pain severity. Interestingly, the pre-to-postoperative differences in the average VAS back and leg pain scores were comparable between patients with and without a reoperation. There were no statistically significant differences between both study subpopulations, with regard to pre- and perioperative clinical data.

To capture all reoperations that were performed at an outside institution, patients were asked about any other related spine surgery after the index microdiscectomy as part of the long-term follow-up questionnaire. Furthermore, since the data collection were completed before hypothesizing a potential difference in the postoperative long-term outcome between both study subpopulations, data acquisition was performed in an unbiased way. In fact, data collection was performed by a coauthor without any conflicts of interest who was not involved in the patient care. Moreover, besides retrospectively collected data, patient reported data were evaluated in the statistical analysis.

The accurate interpretation of the presented findings requires the consideration of the study's limitations. The fact that there was a large proportion of patients who either did not meet the inclusion criteria of the present study or were lost to follow-up represents the main limitation of the present study, due to a potential follow-up bias. To evaluate the potential for bias in the results due to missing data of unavailable patients, we compared demographic and surgical details between the study population ( $n=40)$ and patients who were not available for statistical analysis $(n=163)$. Out of the 163 "not available" patients, basic demographic and surgical details were available for 140 patients. There were no significant differences between the study population and the 140 patients who were not available for analysis in terms of the age at surgery $(p=0.752)$, height $(p=0.626)$, weight $(p=0.468)$, BMI $(p=0.134)$, sex $(p>0.999)$, or microdiscectomy level $(p=0.090)$. There was a statistical difference in terms of the side of the surgical approach (right- vs. left-sided approach) with a $p$ value of 0.046 (not shown). Another limitation of the present study is that not all patients had available VAS scores reported in the medical records, and that missing VAS scores needed to be retrieved from the documented patient history and estimated based on the individual description of the pain severity. Despite the fact that only a small proportion of preoperative VAS scores needed to be estimated (preoperative back and leg pain estimation in $12.5 \%[n=5]$ and $20.0 \%$ [ $n=8$ ] of patients, respectively), and that all pain scores were collected by a single independent co-author without any conflict of interest, the reported mean VAS scores for each subcohort need to be seen as estimated values when interpreting the reported findings. Moreover, the observed postoperative differences in some of the ODI questions between patients with and without a reoperation may be biased by the missing and therefore unknown preoperative ODI scores. 

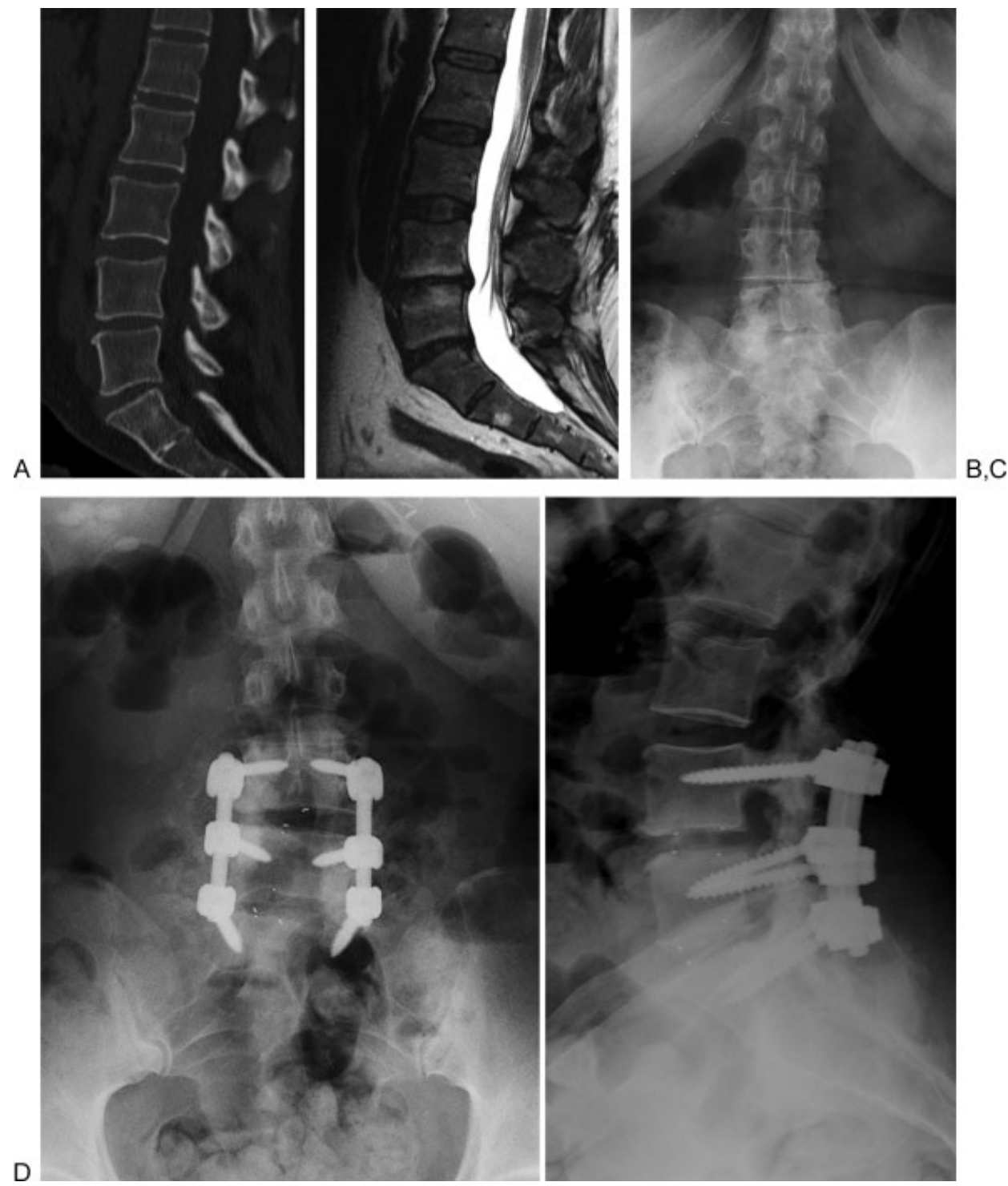

Fig. 3 A 45-year-old female patient presented to the physician office complaining of lower back pain (VAS: 10/10) radiating to the right lower extremity (VAS: 9/10), and a sensory deficit in the L5-S1 distribution. (A) A preoperative computed tomography (CT) imaging study depicted a lumbar disc herniation with central disc protrusion at the L5-S1 level, in addition to a mild retrolisthesis. The patient underwent a single-level microdiscectomy at L5-S1 supplemented by fat-graft placement, and was discharged after 2 days. At her first postoperative office consultation, the symptoms of a sensory deficit in the L5-S1 distribution had resolved, and her back and leg pain had improved, with VAS scores of 4/10 and 1/ 10 , respectively. Due to recurrent radicular symptoms, a magnetic resonance (MR) image was performed 39 months after the index microdiscectomy, which depicted a right paracentral disc herniation at the L5-S1 level, in addition to a severely decreased disc height with posterior bulging of the L4-L5 disc (B) causing mild thecal sac compression, and a slightly progressive right scoliosis with 12 degrees, measured between $\mathrm{L} 1$ and $\mathrm{L} 5(\mathrm{C})$. Forty months after the initial microdiscectomy at $\mathrm{L} 5-\mathrm{S} 1$, the patient underwent a posterior decompression from $\mathrm{L} 4$ to $\mathrm{S} 1$, neurolysis of the L5 and S1 nerve roots, bilateral lateral fusion and posterior instrumentation from L4 to S1, as well as two-level posterior lumbar interbody fusion at L4-L5 and L5-S1 (D). At a postoperative telephone follow-up of 78 months, the reported VAS scores for back and leg pain were both $1 / 10$ with an absence of other radicular symptoms apart from back or leg pain.

Finally, with regard to the subanalysis comparing patients with and without a reoperation after the index microdiscectomy, it needs to be stated that patients in the reoperation subgroup may have a poorer long-term outcome either because they had a reoperation or because they had greater disability or more advanced disease at baseline. In fact, it appears that the reoperation subgroup presented with a trend toward a higher back pain severity already in the preoperative setting. Follow-up studies with available preoperative ODI scores and a larger sample size are warranted to investigate the trend toward a poorer clinical long-term outcome in the reoperation subgroup, as reflected in the ODI score and the degree of patient satisfaction.

\section{Summary and Conclusion}

In conclusion, according to our findings, a minimally invasive approach for the treatment of LDH results in a favorable postoperative long-term outcome in the majority of cases. However, the reoperation rate in our series was higher as previously reported in investigations with a generally 
shorter postoperative follow-up period. Although there were neither pre- nor perioperative statistically significant, clinical differences between patients with and without a reoperation related to the index microdiscectomy, our findings suggest a potential difference with regard to the long-term outcome, as reflected in the ODI score. However, further follow-up studies with available preoperative ODI scores and a larger sample size are warranted to investigate whether these differences were observed because of the performance of an additional lumbar spine surgery or a more advanced disease at baseline in the reoperation subcohort.

\section{Disclosures \\ Alexander Aichmair, none \\ Jerry Y. Du, none \\ Jennifer Shue, none \\ Gisberto Evangelisti, none \\ Andrew A. Sama, none \\ Alexander P. Hughes, none \\ Darren R. Lebl, none \\ Jayme C. Burket, none \\ Frank P. Cammisa, none \\ Federico P. Girardi, none}

Note

This study is IRB approved (IRB\# 12083).

\section{Funding}

There is no funding received for this work from any of the following organizations: National Institutes of Health (NIH); Wellcome Trust; Howard Hughes Medical Institute (HHMI); and other(s).

\section{References}

1 Schiller JS, Lucas JW, Ward BW, Peregoy JA. Summary health statistics for U.S. adults: National Health Interview Survey, 2010. National Center for Health Statistics. Vital Health Stat 10(252);
2012. Available at: http://www.cdc.gov/nchs/data/series/sr_10/ sr10_252.pdf. Accessed May 20, 2013

2 Dagenais S, Caro J, Haldeman S. A systematic review of low back pain cost of illness studies in the United States and internationally. Spine J 2008;8(1):8-20

3 Hart LG, Deyo RA, Cherkin DC. Physician office visits for low back pain. Frequency, clinical evaluation, and treatment patterns from a U.S. national survey. Spine (Phila Pa 1976) 1995;20(1):11-19

4 Govind J. Lumbar radicular pain. Aust Fam Physician 2004;33(6): 409-412

5 Weinstein JN, Lurie JD, Tosteson TD, et al. Surgical vs nonoperative treatment for lumbar disk herniation: the Spine Patient Outcomes Research Trial (SPORT) observational cohort. JAMA 2006;296(20): 2451-2459

6 Goald HJ. Microlumbar discectomy: follow-up of 477 patients. J Microsurg 1980;2(2):95-100

7 Williams RW. Microlumbar discectomy: a conservative surgical approach to the virgin herniated lumbar disc. Spine (Phila Pa 1976) 1978;3(2):175-182

8 Soliman J, Harvey A, Howes G, Seibly J, Dossey J, Nardone E. Limited microdiscectomy for lumbar disk herniation: a retrospective longterm outcome analysis. J Spinal Disord Tech 2014;27(1):E8-E13

9 Porchet F, Bartanusz V, Kleinstueck FS, et al. Microdiscectomy compared with standard discectomy: an old problem revisited with new outcome measures within the framework of a spine surgical registry. Eur Spine J 2009;18(Suppl 3):360-366

10 German JW, Adamo MA, Hoppenot RG, Blossom JH, Nagle HA. Perioperative results following lumbar discectomy: comparison of minimally invasive discectomy and standard microdiscectomy. Neurosurg Focus 2008;25(2):E20

11 Fairbank JC, Couper J, Davies JB, O’Brien JP. The Oswestry low back pain disability questionnaire. Physiotherapy 1980;66(8): 271-273

12 McGirt MJ, Ambrossi GL, Datoo G, et al. Recurrent disc herniation and long-term back pain after primary lumbar discectomy: review of outcomes reported for limited versus aggressive disc removal. Neurosurgery 2009;64(2):338-344, discussion 344-345

13 McGirt MJ, Eustacchio S, Varga P, et al. A prospective cohort study of close interval computed tomography and magnetic resonance imaging after primary lumbar discectomy: factors associated with recurrent disc herniation and disc height loss. Spine (Phila Pa 1976) 2009;34(19):2044-2051

14 Carragee EJ, Han MY, Suen PW, Kim D. Clinical outcomes after lumbar discectomy for sciatica: the effects of fragment type and anular competence. J Bone Joint Surg Am 2003;85-A(1): 102-108

15 Yorimitsu E, Chiba K, Toyama Y, Hirabayashi K. Long-term outcomes of standard discectomy for lumbar disc herniation: a follow-up study of more than 10 years. Spine (Phila Pa 1976) 2001;26(6):652-657 


\section{Editorial Perspective}

This article was accepted after robust discussions among EBSJ reviewers. The authors were able to address the concerns raised and acceptance was granted in light of the thoughtprovoking insights gained by this article with its strengths being long-term follow-up and study of a consistently applied intervention in form of minimally invasive microdiscectomy. The questions posed were simple yet of high interest: longterm outcomes and reoperation rates.

There were two important methodological concerns: Even though this is a retrospective study where the majority of the data came from the medical records, the issue of follow-up bias is just as important. It is not appropriate to have an exclusion criteria that pertains to data collected after a person is treated. For example, missing data or subjects not available for a telephone interview are akin to a subject who is enrolled prospectively that cannot be contacted and hence "lost to follow-up." It is an important distinction. Inclusion/exclusion criteria pertain to the generalizability of the results. Patients who ordinarily would be eligible but did not contribute to the results should be treated separately and unfortunately contribute to the potential for follow-up bias. That is why is it so important to provide the data on the similarities between these groups which was done well in this study. That techni- cally means the follow-up rate will be less than $25 \%$. The problem with follow-up bias is that it may not be random. That is, people who cannot be reached by phone may have been more or less likely to have had a reoperation or more or less likely to have a poor outcome. We will not know for sure.

The other important issue is the comparison between those who have reoperation and those who do not with respect to long-term outcomes. It is not entirely clear if the authors are implying these patients do worse because they had an operation or worse because they had greater disability or more advanced disease as baseline. The data does not support either theory. In fact, it appears that the reoperation group presented with more back pain and there were some other differences like preoperative motor deficit and discectomy levels. Statistical significance is probably less important with such a small sample size. The absolute differences are worth noting and may explain why the reoperation group had less satisfaction as well as lower ODI scores. Without a preoperative ODI score, we, however, have no way of knowing what the follow-up score represents.

EBSJ commends the authors for a worthy effort and being willing to publish less than perfect results to allow us more realistic outcomes prognostication and patient counseling. 